\title{
PDP1ع Functions Downstream of the Circadian Oscillator to Mediate Behavioral Rhythms
}

\author{
Juliana Benito, ${ }^{1 \star}$ Hao Zheng, ${ }^{1,2 \star}$ and Paul E. Hardin ${ }^{2}$ \\ ${ }^{1}$ Department of Biology and Biochemistry, University of Houston, Houston, Texas 77204, and ${ }^{2}$ Center for Research on Biological Clocks, Department of \\ Biology, Texas A\&M University, College Station, Texas 77843
}

\begin{abstract}
The Drosophila circadian oscillator is composed of autoregulatory period/timeless (per/tim) and Clock (Clk) feedback loops that control rhythmic transcription. In the Clk loop, CLOCK-CYCLE heterodimers activate vrille (vri) and PAR domain protein $1 \varepsilon(P d p 1 \varepsilon)$ transcription, then sequential repression by VRI and activation by PDP1 $\varepsilon$ mediate rhythms in $C l k$ transcription. Because VRI and PDP1 $\varepsilon$ bind the same regulatory element, the VRI/PDP1 $\varepsilon$ ratio is thought to control the level of $C l k$ transcription. Thus, constant high or low PDP1 $\varepsilon$ levels in clock cells should eliminate $C l k$ mRNA cycling and disrupt circadian oscillator function. Here we show that reducing PDP1 $\varepsilon$ levels in clock cells by $\sim 70 \%$ via RNA interference or increasing PDP1 $\varepsilon$ levels by $\sim 10$-fold in clock cells does not alter Clk mRNA cycling or circadian oscillator function. However, constant low or high PDP1 $\varepsilon$ levels in clock cells disrupt locomotor activity rhythms despite persistent circadian oscillator function in brain pacemaker neurons that extend morphologically normal projections into the dorsal brain. These results demonstrate that the VRI/PDP1 $\varepsilon$ ratio neither controls $C l k$ mRNA cycling nor circadian oscillator function and argue that PDP1 $\varepsilon$ is not essential for $C l k$ activation. PDP1 $\varepsilon$ is nevertheless required for behavioral rhythmicity, which suggests that it functions to regulate oscillator output.
\end{abstract}

Key words: circadian rhythms; transcription regulation; feedback; Drosophila; behavior; biological clocks

\section{Introduction}

The Drosophila circadian timekeeping mechanism is composed of interlocked period/timeless (per/tim) and Clock (Clk) transcriptional feedback loops (Glossop et al., 1999). These feedback loops are initiated when CLOCK-CYCLE (CLK-CYC) binds E-boxes to activate per, tim, vrille (vri), and PAR domain protein $1 \varepsilon(P d p 1 \varepsilon)$ transcription around midday (for review, see Hardin, 2004, 2005). In the per/tim loop, per and tim mRNAs accumulate to high levels around dusk (Hardin et al., 1990; Sehgal et al., 1995), then PERIOD-TIMELESS (PER-TIM) heterodimers begin to accumulate in the nucleus around midnight and inhibit CLK-CYC binding to E-boxes (Hunter-Ensor et al., 1996; Myers et al., 1996; Darlington et al., 1998; Lee et al., 1999; Yu et al., 2006), thereby repressing per and tim transcription. After PER and TIM degradation during early morning, this inhibition is released, thus enabling another round of CLK-CYC-dependent transcriptional activation.

In the Clk loop, CLK-CYC represses Clk transcription indi-

\footnotetext{
Received June 23, 2006; revised Jan. 19, 2007; accepted Feb. 1, 2007.

This work was supported by National Institutes of Health Grant NS051280 (P.E.H.). We are grateful to Jeff Hall for providing anti-PER antibody, to Mike Young for providing anti-TIM antibody, to Amita Sehgal for providing anti-PDF antibody, and to Paul Taghert for providing anti-PAP antibody. We are indebted to members of the Hardin laboratory for helpful discussions, to Gregg Roman for use of his laboratory, to Dave Sherry and Fanny Ng for help with confocal microscopy, to Elaine McGuffin and the Duke University Model System Genomics Services for transgenic fly generation, and to Lily Bartoszek for editing this manuscript.

J.B. and H.Z. contributed equally to this work.

Correspondence should be addressed to Dr. Paul E. Hardin, Center for Research on Biological Clocks, Department of Biology, Texas A\&M University, College Station, TX 77843. E-mail: phardin@mail.bio.tamu.edu.

D0I:10.1523/JNEUROSCI.4870-06.2007

Copyright $\odot 2007$ Society for Neuroscience $\quad$ 0270-6474/07/272539-09\$15.00/0
}

rectly through VRI, which accumulates in parallel to its mRNA during the late day and binds V/P-boxes to repress Clk transcription (Cyran et al., 2003; Glossop et al., 2003). When PER-TIM inhibits CLK-CYC late at night, vri mRNA and protein levels fall and $C l k$ repression is removed, thus promoting $C l k$ activation. In addition to derepression by PER-TIM, Clk transcription is activated by PDP1 $\varepsilon$ (Cyran et al., 2003). PDP1 1 is one of eight tissuespecific PDP1 isoforms that arise via alternative splicing (Reddy et al., 2000; Grumbling and Strelets, 2006). Loss of $P d p 1$ leads to developmental lethality, with few $P d p 1^{\mathrm{P} 205}$ null mutant animals surviving to the pupal stage (Cyran et al., 2003). In clock cells, $P d p 1 \varepsilon$ mRNA and protein do not accumulate to high levels until late at night (Cyran et al., 2003). Because PDP1 $\varepsilon$ levels rise above those of VRI, PDP1 $\varepsilon$ is thought to displace VRI on V/P-boxes to activate Clk transcription (Cyran et al., 2003). However, Clk mRNA is expressed at peak levels in $C l k^{\mathrm{Jrk}}$ and $c y c^{01}$ mutants (Glossop et al., 1999), suggesting that Clk can be activated independently of PDP1e. This result contrasts with the loss of Clk expression in $P d p 1^{\text {P205 }}$ larvae (Cyran et al., 2003), although it is possible that loss of all PDP1 isoforms causes developmental defects that alter clock cell fate or function, thus indirectly eliminating $C l k$ expression.

If $C l k$ mRNA cycling is driven by competition between PDP1 $\varepsilon$ and VRI for V/P-box binding, then reducing or increasing PDP1 $\varepsilon$ expression in clock cells should disrupt Clk mRNA cycling and, consequently, circadian oscillator function. Here we use the Gal4/UAS system to drastically reduce (via RNA interference) or increase PDP1e levels in oscillator cells. Constant low or high PDP1e levels had little effect on Clk mRNA cycling and abundance, thus demonstrating that the VRI/PDP1 $\varepsilon$ ratio does 
not regulate $C l k$ mRNA rhythms or circadian oscillator function. However, constant low or high PDP1 1 levels drastically reduced behavioral rhythmicity even though oscillator function persists in pacemaker cells that drive rhythmic behavior. These data demonstrate that PDP1 $1 \varepsilon$ is not an essential component of the circadian oscillator, but rather functions to mediate circadian clock output.

\section{Materials and Methods}

PDP1e antibody generation. Full-length PDP1e was expressed in Escherichia coli, purified, and used to produce antisera in guinea pigs. pET28a $(+)$ vector was initially modified to contain an SpeI site by inserting the duplexed $\mathrm{Na}$ and $\mathrm{Ns}$ oligonucleotides $\left(\mathrm{Na}, 5^{\prime}\right.$-GATCCATACTAGTACACGAATTCGTATCCGACGACA-3'; Ns, 5'-TATGTCGTCGGATACGAATTCGTGTACTAGTATG- ${ }^{\prime}$ ) at the NdeI and BamHI sites. An $\sim 2.4 \mathrm{~kb}$ SpeI-XhoI fragment from Pdp1 1 cDNA clone CG27708 was inserted into the modified $\mathrm{pET} 28 \mathrm{a}(+)$ vector to generate pET28a $(+)-P d p 1 \varepsilon$, which expresses an N-terminal HIS-tagged PDP1 $\varepsilon$ fusion protein that contains the entire coding sequence of PDP1 $\varepsilon$. pET28a(+) $-P d p 1 \varepsilon$ was transformed into BL21 cells, and protein expres-

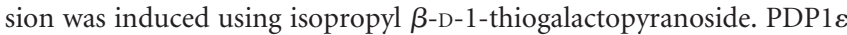
fusion protein was purified from cell lysates using a nickel affinity column (GE Healthcare, Piscataway, NJ) as described previously (Houl et al., 2006). A single band at the predicted molecular weight of the PDP1 $\varepsilon$ fusion protein $(\sim 75 \mathrm{kDa})$ was detected and used to immunize guinea pigs for antibody production (Cocalico Biological, Reamstown, PA). Although the newly generated PDP1 antibody is capable of detecting all eight isoforms of PDP1, PDP1 1 is specifically targeted in clock neurons because this PDP1 isoform is uniquely expressed in the CNS (Reddy et al., 2000; Grumbling and Strelets, 2006) and is eliminated in $C l k^{\mathrm{Jrk}}$ and $c y c^{01}$ mutant flies (see Fig. 1A,B).

Western blotting. The VRI antibody was described previously (Glossop et al., 2003). VRI and PDP $1 \varepsilon$ were detected on $7.5 \%$ polyacrylamide gels. For both antibodies, primary antibodies were used at a dilution of 1:5000 with horseradish peroxidase-conjugated goat anti-guinea pig (Sigma, St. Louis, MO) secondary antibody diluted 1:1000. The relative levels of PDP1 $\varepsilon$ and VRI were quantified as the ratio of PDP1 $1 \varepsilon$ or VRI band intensity to that of a nonspecific background band using Quantity One 1-D Analysis software (Bio-Rad, Hercules, CA). These relative VRI levels were normalized to VRI levels in $w^{1118}$ at Zeitgeber time 17 (ZT17) or ZT15 on each blot to obtain the final levels of VRI.

Fly stocks and transgenic flies. The wild-type (Canton-S), $w\left(w^{1118}\right)$, $c y c^{01}$, per $^{01}$, tim Gal4, and $p d f \mathrm{Gal} 4$ strains have been described previously (Konopka and Benzer, 1971; Hazelrigg et al., 1984; Emery et al., 1998; Rutila et al., 1998; Park et al., 2000). The UAS-PDP1i transgene was constructed as follows. Pdp1 genomic (1200 bp) and cDNA (400 bp) fragments comprising exons 5, 6, and first part of exon 7 were amplified by PCR using primers having unique $5^{\prime}$ restriction sites. Primers used for the genomic fragment were $5^{\prime}$-GAATTCCTGATGGCCTGCCCGGCACGCATCTGG-3' and 5'-GCGGCCGCATTCTGAGCGGA-ATAAATTTGG-3', in which the underlined sequences correspond to EcoRI and NotI sites, respectively. Primers used for the cDNA fragment were 5'-GCGGCCGCATTTTCCTTCTCCAAGTAGCGTGC-3' and 5'GGTACCCTGATGGCCTGCCCGGCACGCATCTGG-3', in which the underlined sequences correspond to NotI and KpnI sites, respectively. When choosing the cDNA fragment for the inverted sequence, special care was taken to avoid splice donor sequences. The cDNA fragment was fused to the genomic fragment in an inverted orientation just after the exon 7 splice acceptor to disrupt direct repeats. Each PCR fragment was gel purified, ligated into pCR-XL-TOPO (Invitrogen, Carlsbad, CA), and transformed following the manufacturer's instructions. Clones were sequenced, and subsequently, the genomic PCR product was excised from pCR-XL-TOPO as an EcoRI-NotI fragment and cloned into the EcoRI and NotI sites of pUAST to form UAS-PdpleGEN. The cDNA fragment was excised using NotI and KpnI and ligated into the NotI and KpnI sites of UAS-Pdp1 $\varepsilon$ GEN to form UAS-PDP1i.

To construct the UAS- $p d p 1 \varepsilon$ transgene, $P d p 1 \varepsilon$ cDNA clone GH27708 was digested with $A v r I I$ and $B s u$ 36I; the resulting fragment was blunted using Klenow and inserted in pBS KS at the EcoRV site to form pBS$P d p 1 \varepsilon$. The $P d p 1 \varepsilon$ cDNA fragment was excised from pBS- $P d p 1 \varepsilon$ using XhoI and NotI and inserted into XhoI and NotI digested pUAST (Brand and Perrimon, 1993) to form UAS-PDP1 $\varepsilon$

The UAS-PDP1i and UAS-PDP1 $\varepsilon$ transgenes were used to generate transgenic flies via P-element-mediated transformation. At least five independent lines were generated for each transgene. Flies with inserts on the second and third chromosome were balanced using In(2LR)CyO or In(3LR)TM6B, respectively. All fly strains were reared on medium containing corn meal, molasses, yeast, agar, and Tegosept (a mold inhibitor) at $25^{\circ} \mathrm{C}$.

RNA quantification. Clk mRNA levels were assayed by quantitative real-time PCR (qPCR). Flies were entrained in a $12 \mathrm{~h}$ light/dark (LD) cycle for at least $3 \mathrm{~d}$, collected every $4 \mathrm{~h}$, and immediately stored at $-80^{\circ} \mathrm{C}$. Total RNA was isolated from fly heads using TRIZOL (Invitrogen) or the ToTALLY RNA kit (Ambion, Austin, TX). To eliminate genomic DNA contamination, each sample was treated with DNase (Promega, Madison, WI). First-strand cDNA was synthesized from $1 \mu \mathrm{g}$ of RNA using random primers and Superscript II (Invitrogen). For qPCR, TaqMan assays were performed using the following Clk primers and probes: forward primer, $5^{\prime}$-GCGTCCCGCTTCGAGTT-3'; reverse primer, 5'-TGGCCTTCGGCGATATTC-3'; probe 6FAM-TGGCAATATCAGCTCCACA-MGBNFQ. Ribosomal protein 49 (RP49) was used as the internal loading control. The primers and TaqMan probe for RP49 were as follows: forward primer, $5^{\prime}$-CTGCCCACCGGATTCAAG3'; reverse primer, 5'-CGATCTCGCCGCAGTAAAC-3'; probe VICCCTCCAGCTCGCGCACGTTG-MGBNFQ. Reactions were run on an Applied Biosystems (Foster City, CA) Prism 7000. The relative levels of $C l k$ and RP49 RNAs were calculated based on standard curves for $C l k$ and RP49 that were run in each assay. Clk levels were normalized to RP49 at each time point.

Brain whole-mount immunofluorescence. Flies were entrained in LD cycles for at least $3 \mathrm{~d}$ and kept in constant darkness (DD). On the second day of DD, transheterozygous UAS-PDP1i+timGal4 and control $w$ flies were collected at circadian time 9 (CT9) and CT21, and their brains were dissected in $1 \times$ PBS, pH 7.4. Brains were fixed with $3.7 \%$ formaldehyde for $15 \mathrm{~min}$ at room temperature, followed by two washes with $1 \times$ PBS and one wash with PBT ( $1 \times$ PBS, pH 7.4, and $0.5 \%$ Triton X-100). Brains were then incubated with $5 \%$ goat serum in PBT for $3 \mathrm{~h}$ at room temperature to reduce nonspecific immunoreactivity. Primary antibodies were diluted in PBT plus $2 \%$ goat serum and incubated with samples at $4{ }^{\circ} \mathrm{C}$ for $48 \mathrm{~h}$. The primary antibodies used were rabbit polyclonal anti-pigment dispersing factor (PDF) (diluted 1:1000; kindly provided by Dr. A. Sehgal, University of Pennsylvania Medical School, Philadelphia, PA) and rat polyclonal anti-TIM (diluted 1:800; kindly provided by Dr. M. W. Young, Rockefeller University, New York, NY). Brains were then equilibrated to room temperature for $2 \mathrm{~h}$ and washed with PBT for $30 \mathrm{~min}$ six times at room temperature. Specimens were incubated overnight with fluorescently labeled secondary antibodies diluted 1:200 in PBT at $4^{\circ} \mathrm{C}$. The secondary antibodies used were $\mathrm{Cy} 3$-conjugated goat anti-rabbit (Jackson ImmunoResearch, West Grove, PA) for anti-PDF and Alexa 488-conjugated goat anti-rat (Invitrogen, Eugene, OR) for anti-TIM. Brains were equilibrated to room temperature for $2 \mathrm{~h}$, washed with PBT for $30 \mathrm{~min}$ six times at room temperature, and mounted on glass slides using Vectashield (Vector Laboratories, Burlingame, CA). This procedure was also used for immunostaining UAS-PDP1i+timGal4 and control UAS-PDP1i flies, except flies were collected at ZT9 and ZT21; PDP1 antiserum was diluted 1:30,000; and PDP1 was detected using an Alexa 488-conjugated goat anti-guinea pig secondary antibody (Invitrogen).

A similar protocol was used to analyze transheterozygous UASPDP $1 \varepsilon+$ tim Gal4 and control UAS-PDP1 $\varepsilon$ flies with the following changes: flies were collected at CT1 and CT13 on the second day of DD; PBT was replaced by PAXD (5\% BSA, 0.3\% Triton X-100, and $0.3 \%$ sodium deoxycholate); primary antibodies were incubated overnight and washed for $1 \mathrm{~h}$ six times; the primary antibodies were rabbit polyclonal anti-PER (diluted 1:15,000; a gift from J. Hall, Brandeis University, Waltham, MA) and guinea pig anti-PAP (diluted 1:1000; a gift from P. Taghert, Washington University, St. Louis, MO) (to detect PDF); secondary antibodies were incubated overnight at $4^{\circ} \mathrm{C}$ and washed for $1 \mathrm{~h}$ six 


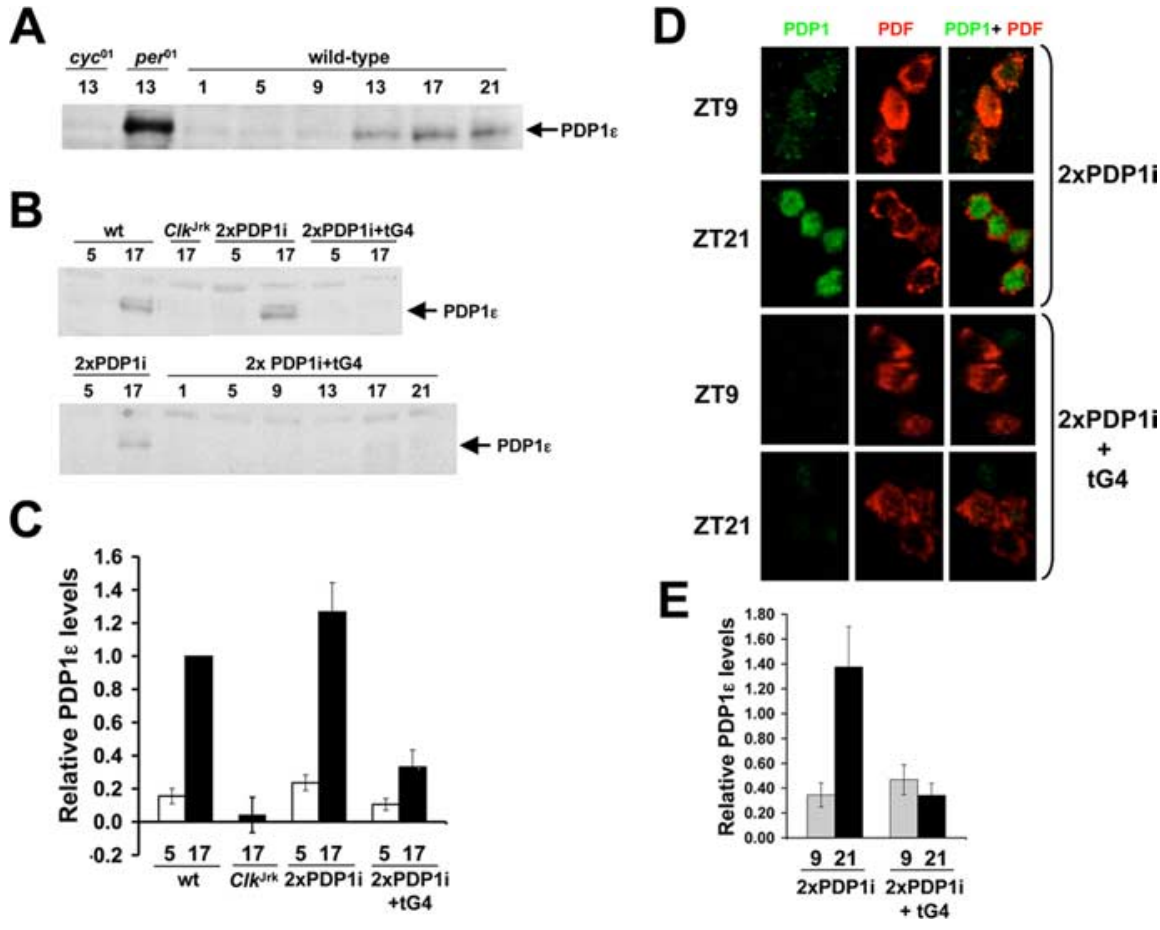

Figure 1. RNAi directed against PDP1 effectively reduces PDP1 $\varepsilon$ levels. $\boldsymbol{A}$, Western blot of head extracts from $c y c^{01}$, per ${ }^{01}$, and wild-type flies collected at the indicated times under LD conditions and probed with PDP1 antibody. $\boldsymbol{B}$, Western blots containing head extracts from wild-type (wt), Clk ${ }^{\text {Jrk }}$, w;UAS-PDP1i/+;UAS-PDP1i/+ (2xPDP1i), and w;UAS-PDP1i/+;UAS-PDP1i/timGal4 (2xPDP1 $+\mathrm{tG4}$ ) flies (top) or 2xPDP1i and 2xPDP1i + tG4 flies (bottom) collected at the indicated times under LD conditions and probed with PDP1 antibody. The Western blot at the top contains a different transheterozygous combination of 2xUAS-PDP1i transgenes than that at the bottom. $\boldsymbol{C}$, Quantification of PDP1 $\varepsilon$ levels in $\boldsymbol{B}$ and at least two independent repeats of each transheterozygous combination. PDP1 $\varepsilon$ levels were quantified as described in Materials and Methods. $\boldsymbol{D}$, Brains dissected from 2xPDP1 $+\mathrm{tG} 4$ and control 2xPDP1i flies collected at ZT9 and ZT21 were probed with PDP1 and PDF antibodies. A representative 1 $\mu \mathrm{m}$ optical section of $s L N_{v} s$ is visualized for PDP1 (left panels), PDF (middle panels), or PDP1 plus PDF (right panels). E, PDP1 immunostaining in 2xPDP1 + tG4 and control 2xPDP1 1 flies was quantified as the ratio of PDP1/PDF staining. Staining in $s L N_{v} s$ was quantified from at least nine brains (i.e., at least 3 brains from 3 independent experiments).

times; and the secondary antibodies used were Alexa 488-conjugated goat anti-rabbit (to detect PER) and Cy3-conjugated goat anti-guinea pig (to detect PDF). This procedure was also used for immunostaining UASPDP1 1 + timGal4 and control UAS-PDP1 $\varepsilon$ flies, except flies were collected at ZT9 and ZT21; PDP1 antiserum was diluted 1:30,000; PDP1 was detected using an Alexa 488-conjugated goat anti-guinea pig secondary antibody (Invitrogen); and rabbit polyclonal anti-PDF (kindly provided by Dr. A. Sehgal) was used to detect PDF.

Immunostaining in brains was visualized on an Olympus (Tokyo, Japan) FV1000 confocal microscope. Serial Z-stack images were obtained at $1 \mu \mathrm{m}$ intervals using a $60 \times$ objective. Images were processed using Photoshop (Adobe Systems, San Jose, CA). Immunofluorescence was quantified in the $\mathrm{LN}_{\mathrm{v}}$ s using NIH Image J (version 1.33u; Wayne Rasband, freely available at http://rsb.info.nih.gov/ij/). Briefly, for each group of neurons, mean pixel intensity was calculated for the green (TIM, PER, or PDP1) and red (PDF) channels, respectively. TIM, PER, or PDP1 immunofluorescence was expressed as a ratio of TIM/PDF, PER/PDF, or PDP1/PDF mean pixel intensity, respectively.

To visualize projections from $\mathrm{sLN}_{\mathrm{v}} \mathrm{s}$ and $\mathrm{LLN}_{\mathrm{v}} \mathrm{s}$, brains were dissected from flies collected at ZT21, fixed with 5\% paraformaldehyde for $30 \mathrm{~min}$, and incubated with a 1:800 dilution of rabbit polyclonal anti-PDF for $48 \mathrm{~h}$ at $4^{\circ} \mathrm{C}$. Brains were then washed in PBT six times for $30 \mathrm{~min}$, incubated in a 1:200 dilution of $\mathrm{Cy} 3$-conjugated anti-rabbit secondary antibody for $24 \mathrm{~h}$ at $4^{\circ} \mathrm{C}$, and washed again in $1 \times$ PBST six times for 30 min before being visualized on an Olympus FV1000 confocal microscope. Serial Z-stack images were obtained at $2.5 \mu \mathrm{m}$ intervals using a $20 \times$ objective. Images were processed using Photoshop (Adobe Systems).

Analysis of behavioral rhythms. Locomotor activity of adult male $w$, transheterozygous UAS-PDP1i, transheterozygous UAS-PDP1i+timGal4, transheterozygous UAS-PDP1i+ $p d f \mathrm{Gal} 4$, transheterozygous UAS-PDP1 $\varepsilon$, transheterozygous UAS-PDP1 $1+$ timGal4, and transheterozygous UASPDP $1 \varepsilon+p d f$ Gal 4 flies that were $<3$ d old were monitored as described previously (Tanoue et al., 2004). Briefly, flies were entrained in LD cycles at $25^{\circ} \mathrm{C}$ for $3 \mathrm{~d}$ and transferred into DD. Locomotor activity behavior was monitored continuously during entrainment and for at least $7 \mathrm{~d}$ in DD using Drosophila activity monitors (Trikinetics, Waltham, MA). Data collected during DD were subjected to periodogram analysis using the Fly Activity Analysis Suite for Mac OSX (FaasX, version 0.7.1, copyright 2002-2003; M. Boudinot, Centre National de la Recherche Scientifique-Institut Neurobiologie Alfred Fessard, Gif sur Yvette, France). Flies with a power $\geq 10$ and a width $\geq 2$ in periodogram analysis were designated as rhythmic. Activity was calculated as the number of activity events occurring every $30 \mathrm{~min}$.

\section{Results \\ Constant low PDP1e levels do not disrupt $C l k$ mRNA cycling or oscillator function}

Because VRI-dependent repression and PDP1 $\varepsilon$-dependent activation are thought to control Clk mRNA cycling, we hypothesized that lowering PDP1 $\varepsilon$ levels would disrupt $C l k$ mRNA cycling by reducing $C l k$ mRNA levels and/or increasing the proportion of the cycle that $C l k$ is at trough levels. To test this hypothesis, we used the UAS/GAL4 system to inhibit PDP1 expression in clock cells via RNA interference. A PDP1 RNA interference transgene (UAS-PDP1i) was generated and driven in clock cells by tim Gal4. Flies bearing both the UAS-PDP1i and timGal4 transgenes, which we will refer to as UAS-PDP1i+ tim Gal4, were entrained in LD cycles along with UAS-PDP1iand $w$-positive control and $C l k^{\text {Jrk }}$-negative control flies, collected at times when PDP1 $\varepsilon$ levels are high (ZT17, where ZT 0 refers to lights on and ZT12 refers to lights off during LD) or low (ZT5) in wild-type flies (Cyran et al., 2003), and used to prepare head extracts for Western blot analysis. Western blot analyses were probed with a newly generated antibody that detects PDP $1 \varepsilon$ based on the following criteria: it recognizes a $\sim 80 \mathrm{kDa}$ protein that is rhythmically expressed with a peak around ZT17-ZT21, it is absent in $C l k^{\mathrm{Jrk}}$ and $c y c^{01}$ flies, and it is at high levels in per ${ }^{01}$ flies (Cyran et al., 2003) (Fig. 1 A,B). Flies with transheterozygous UAS-PDP1i inserts driven by timGal4, which we will refer to as $2 \times P D P 1 i+t i m G a l 4$, reduce PDP $1 \varepsilon$ levels to $\sim 25-$ $30 \%$ of control $2 \times \mathrm{PDP} 1 \mathrm{i}$ and $w$ values at ZT17, which is close to normal PDP $1 \varepsilon$ trough levels (Fig. $1 B, C$ ). These low levels of PDP $1 \varepsilon$ are not attributable to a phase shift in $2 x \mathrm{xAS}$ PDP1i+tim Gal4 flies because PDP1 $\varepsilon$ remains at low levels in head extracts from $2 x P D P 1 i+t i m G a l 4$ flies collected every $4 \mathrm{~h}$ (Fig. $1 B$ ). These results establish that RNA interference directed against $P d p 1$ effectively inhibits PDP1 $1 \varepsilon$ expression in heads from 2xUAS-PDP $1 \mathrm{i}+$ tim Gal4 flies.

Because $>80 \%$ of clock gene expression in fly heads emanates from photoreceptors (Glossop and Hardin, 2002), we wanted to determine whether low levels of PDP1 $1 \varepsilon$ are also seen in brain 
pacemaker neurons from $2 \times \mathrm{xDP} 1 \mathrm{i}+$ tim Gal4 flies. 2xPDP1i+tim Gal4 and control 2xPDP1i flies were entrained in LD cycles and collected at times when PDP1 $\varepsilon$ levels are high (ZT21) or low (ZT9). Brains from these flies were dissected, probed with antisera against PDP1 $\varepsilon$ and the $\mathrm{LN}_{\mathrm{v}}$ marker PDF, and imaged via confocal microscopy. In brains from $2 x P D P 1 i$ flies, the intensity of PDP $1 \varepsilon$ immunofluorescence in $\mathrm{LN}_{\mathrm{v}} \mathrm{s}$ was high at $\mathrm{ZT} 21$ and low at ZT9, whereas the intensity of PDP1 $\varepsilon$ immunofluorescence in $\mathrm{LN}_{\mathrm{v}} \mathrm{s}$ from 2xPDP1i+timGal4 brains remained low at both ZT21 and ZT9 (Fig. 1D,E). These results demonstrate that RNA interference directed against $P d p 1$ effectively inhibits PDP $1 \varepsilon$ expression in $\mathrm{LN}_{\mathrm{v}} \mathrm{s}$ from $2 \mathrm{xUAS}-$ PDP1i+timGal4 flies.

To determine whether low levels of PDP $1 \varepsilon$ disrupt $C l k$ mRNA cycling, we entrained 2xUAS-PDP1i+timGal4 and control tim Gal4 flies, collected them every $4 \mathrm{~h}$ starting at ZT1, and extracted head mRNA for qPCR analysis. Clk mRNA in 2xUASPDP1i+timGal4 flies cycled with an approximate fivefold amplitude (Fig. $2 A$ ), which is similar to that in tim Gal4 control (Fig. 2) and wild-type flies (Bae et al., 1998; Darlington et al., 1998). Clk mRNA was also measured in 2xUAS-PDP1i flies; however, an $\sim 10$-fold Clk mRNA cycling was seen (data not shown), mainly because of anomalously high peak levels. The waveform of $\mathrm{Clk}$ mRNA cycling in 2xUAS-PDP1i+timGal4 and control timGal4 flies was similar, with Clk mRNA accumulating to peak levels around dawn and falling to trough levels around dusk (Fig. 2A). Just after dawn, Clk mRNA levels in 2xUAS-PDP1i+timGal4 flies are somewhat lower than in control tim Gal4 flies, which might suggest some effect of PDP $1 \varepsilon$ in lowering Clk mRNA levels at this time. Nevertheless, these results demonstrate that reduced PDP1 $\varepsilon$ levels have relatively little effect on Clk mRNA cycling amplitude and waveform.

The approximate fivefold cycling of Clk mRNA in 2xUASPDP1i+timGal4 flies suggests that the circadian feedback loops are functioning normally. Because VRI functions to repress $\mathrm{Clk}$ transcription, we would expect to see high-amplitude VRI cycling that is antiphase to that of Clk mRNA (Cyran et al., 2003; Glossop et al., 2003). Indeed, VRI levels increase as Clk mRNA levels decrease, and $C l k$ mRNA levels increase as VRI levels decrease in 2xUAS-PDP1i+timGal4 flies (Fig. 2B,C). This result is consistent with the role of VRI as a $C l k$ repressor and provides further confirmation that the circadian feedback loop is functional in 2xUAS-PDP1i+timGal4 flies. The high VRI/PDP1e ratio in 2xUAS-PDP1i+timGal4 flies would predict that Clk mRNA should be quite low, yet $C l k$ mRNA levels and cycling are not appreciably altered. This result indicates that VRI/PDP $1 \varepsilon$ ratio does not control Clk mRNA cycling and argues that Clk may be activated by factors other than PDP1 $1 \varepsilon$.

\section{Constant high PDP1 $\varepsilon$ levels do not disrupt $C l k$ mRNA cycling or oscillator function}

If PDP $1 \varepsilon$ activates $C l k$ transcription, we would expect that constant high levels of PDP1 $\varepsilon$ would decrease the VRI/PDP1 $\varepsilon$ ratio, resulting in higher $C l k$ mRNA levels and a reduced Clk mRNA cycling amplitude. To determine whether this is the case, we used

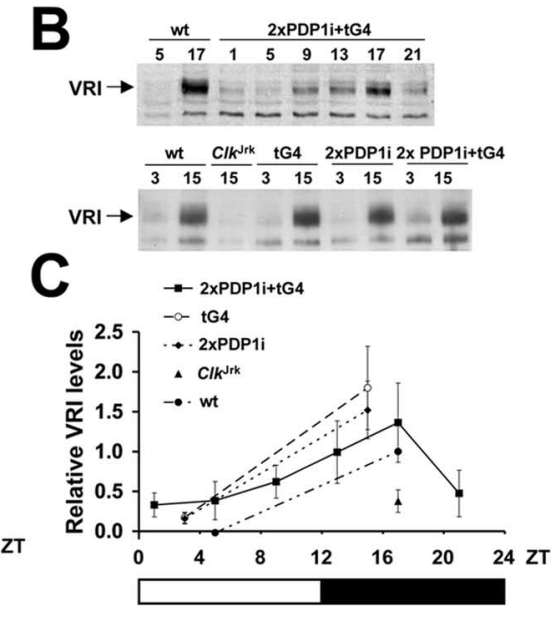
timGal4/+ (timGal4) and w;UAS-PDP1i/+ +UAS-PDP1i/timGal4 (2xPDP1i + tG4) flies collected at the indicated times under LD conditions (ZT). Relative Clk mRNA levels were quantified as described in Materials and Methods. The black and white bars ied as described in Materials and Methods and normalized to wild type at ZT17. wt, Wild type.

the UAS/GAL4 system to overexpress PDP1 $\varepsilon$ in clock cells. A UAS-PDP $1 \varepsilon$ transgene was constructed and driven in clock cells by timGal4. Flies containing both the UAS-PDP1 $\varepsilon$ and timGal4 transgenes, which we will refer to as UPDP $1 \varepsilon+$ timGal4 flies, were entrained in $\mathrm{LD}$ cycles along with control flies containing the UAS-PDP $1 \varepsilon$ insert only (UPDP $1 \varepsilon$ ), collected every $6 \mathrm{~h}$ during LD starting at ZT3, and used to prepare head extracts for Western blot analysis. Flies with two different UPDP1 $1 \varepsilon$ inserts driven by timGal4 have between 5- and 20-fold higher levels of PDP1 $\varepsilon$ compared with control UPDP $1 \varepsilon$ flies, depending on the time point (Fig. $3 A, B$ ), demonstrating that UPDP $1 \varepsilon+$ tim Gal4 flies do indeed overexpress PDP $1 \varepsilon$ in fly heads.

To determine whether UPDP $1 \varepsilon+$ tim Gal4 flies also produce high levels of PDP1 $1 \varepsilon$ in brain pacemaker neurons, UPDP $1 \varepsilon+$ tim Gal 4 and UPDP1 $\varepsilon$ control flies were entrained in LD cycles and collected at times when PDP1 $\varepsilon$ levels are high (ZT21) or low (ZT9). Brains from these flies were dissected, probed with PDP $1 \varepsilon$ and PDF antisera, and imaged via confocal microscopy. In brains from UPDP1 $\varepsilon$ flies, the intensity of PDP1 $1 \varepsilon$ immunofluorescence was high at ZT21 and low at ZT9 in $\mathrm{LN}_{\mathrm{v}} \mathrm{s}$, whereas the intensity of PDP1 1 immunofluorescence in $\mathrm{LN}_{\mathrm{v}}$ s from UPDP $1 \varepsilon+$ tim Gal4 brains remained high at both ZT21 and ZT9 (Fig. 3C,D). Thus, consistent with results in whole heads, PDP1 $\varepsilon$ expression is constantly high in $\mathrm{LN}_{\mathrm{v}} \mathrm{s}$ of UPDP1 $1 \varepsilon+$ timGal4 flies.

To determine whether high levels of PDP1 $\varepsilon$ disrupt $C l k$ mRNA cycling, we entrained UPDP $1 \varepsilon+$ tim $\mathrm{Gal} 4$ flies and control UPDP $1 \varepsilon$ flies in LD, collected them every $4 \mathrm{~h}$ starting at ZT1, and extracted head mRNA for qPCR analysis. The overall levels of $\mathrm{Clk}$ mRNA in UPDP $1 \varepsilon+$ tim Gal4 flies were comparable to those in control UPDP1 $\varepsilon$ flies (Fig. 4A). The phase of Clk mRNA cycling was also similar in UPDP1 $\varepsilon+$ tim Gal4 and control UPDP1 $\varepsilon$ flies in that the peak was somewhat variable (i.e., between ZT21 and ZT5 depending on the strain) and the trough was at ZT13 (Fig. $4 A$ ). From these results, it is apparent that high levels of PDP1 $\varepsilon$ neither increased the overall levels of Clk mRNA nor broadened the Clk mRNA peak, indicating that the VRI/PDP1 $\varepsilon$ ratio does not control Clk mRNA cycling.

Cycling of $C l k$ mRNA in UPDP1 $1 \varepsilon+$ tim Gal4 flies suggests that 


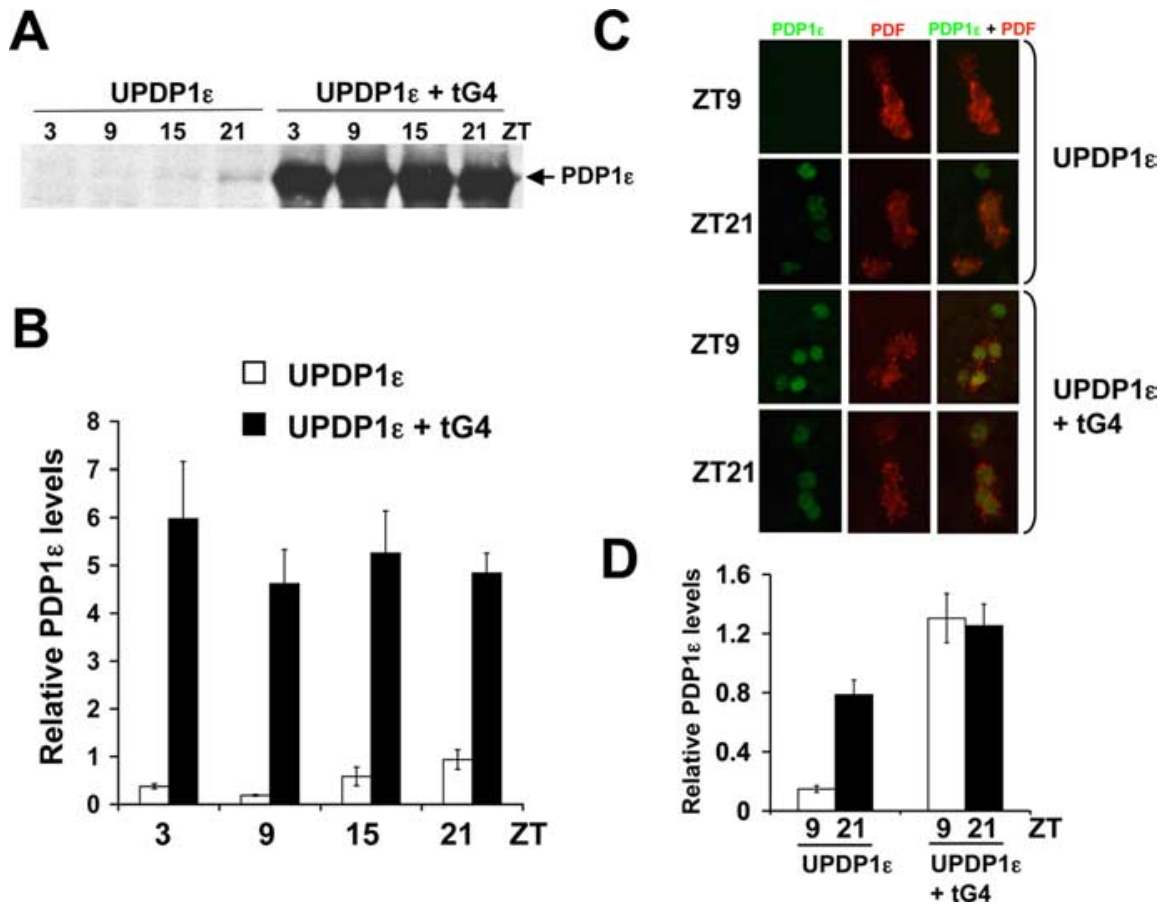

Figure 3. Overexpression of PDP1e in oscillator cells. $A$, Western blot of head extracts from w;UAS-PDP1 $1 \varepsilon /+;+/+(U P D P 1 \varepsilon)$ and $\mathrm{w} ; \mathrm{UAS}-\mathrm{PDP} 1 \varepsilon /+;$ timGal4/+ (UPDP1 $\varepsilon+\mathrm{tG} 4)$ flies collected at the indicated times under $\mathrm{LD}$ conditions and probed with PDP1 antibody. B, Quantification of PDP1 $\varepsilon$ levels in $\boldsymbol{A}$ and at least four independent repeats using three different UAS-PDP1 $\varepsilon$ inserts. PDP1 $\varepsilon$ levels were quantified as described in Materials and Methods. C, Brains dissected from UPDP1 $\varepsilon+\mathrm{tG} 4$ and control UPDP1 $\varepsilon$ flies collected at ZT9 and ZT21 were probed with PDP1 and PDF antibodies. A representative $1 \mu$ m optical section of $s L N_{v} s$ is visualized for PDP1 (left panels), PDF (middle panels), or PDP1 plus PDF (right panels). D, PDP1 immunostaining in UPDP1 $\varepsilon+\mathrm{tG} 4$ and control UPDP1 $\varepsilon$ flies was quantified as the ratio of PDP1/PDF staining. Staining in $\mathrm{LLN}_{v} s$ was quantified from at least four brains (i.e., at least 2 brains from 2 independent experiments).

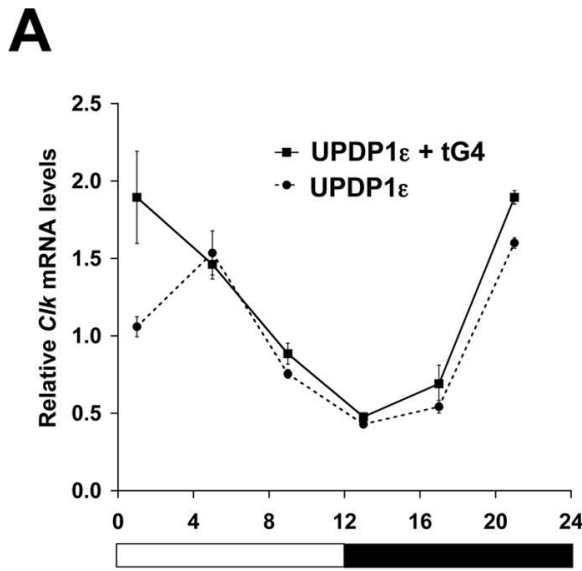

Zeitgeber Time (hr)

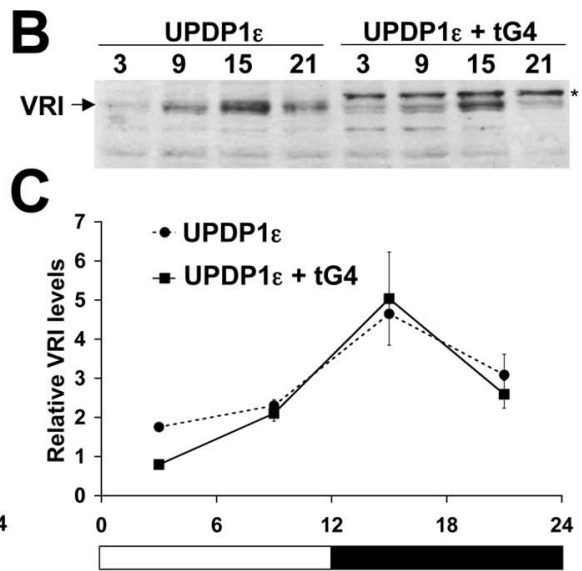

Zeitgeber Time (hr)

Figure 4. Overexpression of PDP1 $1 \varepsilon$ in oscillator cells does not disrupt $C l k m R N A$ or VRI cycling. A, qPCR of Clk mRNA from the heads of $w ;$ UAS-PDP1 $1 \varepsilon /+; U A S-P D P 1 \varepsilon /+(U P D P 1 \varepsilon)$ and $w$; UAS-PDP1 $1 \varepsilon /+; U A S-P D P 1 \varepsilon / t i m G a l 4$ (UPDP1 $\varepsilon+$ tG4) flies collected at the indicated times under LD conditions (ZT). Relative CIk mRNA levels were quantified as described in Materials and Methods. The black and white bars represent times when lights were on or off, respectively. $\boldsymbol{B}$, Western blot of head extracts from UPDP1 $\varepsilon$ and UPDP1 $\varepsilon+$ tG 4 flies collected at the indicated times under LD conditions and probed with VRI antibody. The asterisk denotes the PDP1 $\varepsilon$ band that did not wash off completely from the blot in Figure 3A. C, Quantification of VRI levels in the Western blot in $\boldsymbol{B}$ and at least two independent repeats. VRI levels were quantified as described in Materials and Methods.

the circadian oscillator is functional and predicts that VRI should cycle in the opposite phase as Clk mRNA (Cyran et al., 2003; Glossop et al., 2003). To determine whether this is the case, VRI levels were measured in UPDP1 1 + tim Gal 4 and control UPDP1 $\varepsilon$ flies under LD conditions. The same blot used to show that
PDP1e was overexpressed in UPDP $1 \varepsilon+$ timGal4 flies (Fig. 3A) was reprobed with VRI antiserum to reveal a rhythm in VRI levels similar to that in control flies (Fig. 4B,C). Peak levels of VRI coincided with trough levels of $C l k$ mRNA, as would be expected given that VRI represses Clk transcription (Cyran et al., 2003; Glossop et al., 2003). These data also confirm that UPDP1 $1 \varepsilon+$ tim Gal4 flies contain a functional circadian oscillator.

Constant high or low PDP1e levels disrupt behavioral rhythms without disrupting oscillator function in brain pacemaker neurons

Given that constant high or constant low levels of PDP1e had little or no effect on circadian oscillator function, we expected that altered PDP1e levels would not affect locomotor activity rhythms. Locomotor activity of flies expressing constant low (2xUAS-PDP1i+timGal4, 2xUAS$\mathrm{PDP} 1 \mathrm{i}+p d f \mathrm{Gal} 4)$ or high (2xUAS$\mathrm{PDP} 1 \varepsilon+$ timGal4, 2xUAS-PDP1 $\varepsilon+p d f \mathrm{Gal} 4$ ) PDP1 $\varepsilon$ levels was measured along with control (2xUAS-PDP1i, 2xUAS-PDP1 $\varepsilon$, timGal4, and pdfGal4) flies under DD conditions after $3 \mathrm{~d}$ of entrainment in LD cycles. Surprisingly, only $\sim 17 \%$ of flies expressing constant low levels of PDP1 $1 \varepsilon$ in clock cells (i.e., 2xUAS-PDP1i+timGal4) displayed rhythms in locomotor activity compared with $\sim 78 \%$ of UAS-PDP1i transgene only and $\sim 80 \%$ of timGal 4 driver only control flies (Table 1). Rhythmic 2xUASPDP1i+timGal4 flies had long period rhythms with a lower power than controls. Overall activity was somewhat lower in 2xUAS-PDP1i+timGal4 flies than $w$ and timGal4 controls, whereas 2xUAS-PDP1i controls had anomalously high levels of activity. When PDP1i expression was restricted to $\mathrm{LN}_{\mathrm{v}} \mathrm{s}$ using the $p d f \mathrm{Gal} 4$ driver (i.e., 2xUAS-PDP1i+pdfGal4), locomotor activity rhythms were abolished, whereas $\sim 57 \%$ of control pdfGal4 flies displayed rhythmic locomotor activity (Table 1). Overall activity of 2xUAS-PDP1i+pdfGal4 flies was comparable to 2xUAS-PDP1i+ timGal4 flies.

PDP1 $1 \varepsilon$ overexpression also disrupted locomotor activity rhythms. Only $\sim 12 \%$ of flies that constantly overexpress PDP $1 \varepsilon$ in all clock cells (i.e., 2xUAS-PDP1 $1+$ timGal4) exhibited rhythmic locomotor activity compared with $\sim 74 \%$ of $2 x U A S-$ PDP1 $\varepsilon$ flies and $100 \%$ of timGal4 flies (Table 1). Restricting PDP1 $\varepsilon$ expression to $\mathrm{LN}_{\mathrm{v}} \mathrm{s}$ using $p d f \mathrm{Gal} 4$ resulted in $\sim 10 \%$ of flies having rhythmic locomotor activity versus $\sim 95 \%$ of $p d f G a l 4$ control flies (Table 1). The power of rhythmic 2xUAS-PDP1 1 +timGal 4 and $2 \mathrm{xUAS}-\mathrm{PDP} 1 \varepsilon+p d f \mathrm{Gal} 4$ flies was lower than $2 \mathrm{xUAS}-\mathrm{PDP} 1 \varepsilon$, tim- 
Gal4, and $w$ controls. Overall activity levels in $2 x U A S-P D P 1 \varepsilon+$ timGal 4 and $2 \mathrm{xUAS}-\mathrm{PDP} 1 \varepsilon+p d f \mathrm{Gal} 4$ flies was similar to $w$, timGal4, and $p d f \mathrm{Gal} 4$ flies, whereas 2xUAS-PDP1 $\varepsilon$ flies had anomalously high activity levels. These results demonstrate that constant high or low levels of PDP1E severely disrupt locomotor activity rhythms. However, circadian oscillator function persists in flies with constant high or low PDP1e levels, which suggests that PDP1e affects locomotor activity rhythms as an output of the circadian clock rather than as a component of the clock.

Although the circadian oscillator continues to operate in fly heads when PDP $1 \varepsilon$ is constantly high or low, this does not necessarily mean that circadian oscillators are functioning in the pacemaker cells that control locomotor activity rhythms because photoreceptors represent $>80 \%$ of circadian oscillators in fly heads (Glossop and Hardin, 2002). To determine whether flies expressing constant low levels of PDP $1 \varepsilon$ have functional $\mathrm{LN}_{\mathrm{v}}$ oscillators, the abundance of TIM was assessed in $\mathrm{LN}_{\mathrm{v}} \mathrm{s}$ via immunostaining. UASPDPli+timGal4 and control $w$ flies were entrained to LD cycles and collected at CT21 and CT9 on the second day of DD. Brains from these flies were dissected and probed with antisera against TIM and the $\mathrm{LN}_{\mathrm{v}}$ marker PDF and imaged via confocal microscopy. In both $2 x U A S-$ PDP1i+timGal 4 and control $w$ brains, the intensity of TIM immunofluorescence was high at CT21 and low at CT9 in $\mathrm{LN}_{\mathrm{v}} \mathrm{s}$ (Fig. 5). To assess $\mathrm{LN}_{\mathrm{v}}$ oscillator function in flies expressing constant high levels of PDP $1 \varepsilon, 2 \mathrm{xUAS}-\mathrm{PDP} 1 \varepsilon+$ timGal4 and control $w$ flies were entrained to LD cycles and collected at CT1 and CT13 on the second day of DD. Brains from these flies were dissected and probed with antisera against PER and PDF and imaged via confocal microscopy. In both 2xUASPDP $1 \varepsilon+$ tim Gal4 and control $w$ brains, the intensity of PER immunofluorescence was high at CT1 and low at CT13 in $\mathrm{LN}_{\mathrm{v}} \mathrm{s}$ (Fig. 6). Rhythmic expression of TIM and PER in $\mathrm{LN}_{\mathrm{v}} \mathrm{s}$ from $2 \mathrm{xUAS}-\mathrm{PDP} 1 \mathrm{i}+$ tim $\mathrm{Gal}$ 4 and $2 x U A S-P D P 1 \varepsilon+$ timGal 4 flies, respectively, demonstrates that locomotor activity pacemaker neurons contain functional circadian oscillators.

Because some behaviorally arrhythmic clock gene variants show molecular rhythmicity over the first $2 \mathrm{~d}$ in DD, TIM and PER cycling were assayed in the $\mathrm{LN}_{\mathrm{v}} \mathrm{s}$ of flies expressing reduced or increased levels of PDP1 $\varepsilon$ in clock cells during day 5 in DD. Rhythmic TIM immunofluorescence
Table 1. Reducing and increasing PDP1 $\varepsilon$ levels disrupt locomotor activity rhythms

\begin{tabular}{|c|c|c|c|c|}
\hline Genotype & Percentage rhythmic $(n)$ & Period $(\mathrm{h}) \pm$ SEM & Power \pm SEM & Activity \pm SEM \\
\hline$w ;+/+;+/+$ & $75.0(172)$ & $23.8 \pm 0.03$ & $78.9 \pm 3.7$ & $17.8 \pm 0.6$ \\
\hline w;+/+;tim-Gal4/+ & $80.5(41)$ & $24.6 \pm 0.07$ & $76.7 \pm 5.4$ & $16.7 \pm 0.9$ \\
\hline $\mathrm{w} ;+/+; p d f-G a l 4 /+$ & $57.0(35)$ & $24.2 \pm 0.13$ & $68.7 \pm 7.7$ & $14.0 \pm 0.8$ \\
\hline w;PDP1iA/+;w;PDP1iB/+ & $77.8(38)$ & $23.4 \pm 0.08$ & $72.8 \pm 5.3$ & $28.3 \pm 2.1$ \\
\hline w;PDP1iA/+;w;PDP1iB/tim-Gal4 & $17.2(29)$ & $25.3 \pm 0.23$ & $35.3 \pm 1.7$ & $13.5 \pm 1.0$ \\
\hline w;PDP1iA/+ ; w;PDP1iB/pdf-Gal4 & $0(27)$ & n.a. & n.a. & $12.1 \pm 0.7$ \\
\hline$w ;+/+;+/+$ & $96.0(25)$ & $23.5 \pm 0.15$ & $96.1 \pm 6.4$ & $19.9 \pm 2.3$ \\
\hline w;+/+;tim-Gal4/+ & $100.0(31)$ & $24.3 \pm 0.15$ & $78.4 \pm 5.2$ & $21.6 \pm 2.0$ \\
\hline w;+/+;pdf-Gal4/+ & $95.5(22)$ & $24.3 \pm 0.09$ & $76.4 \pm 9.5$ & $13.5 \pm 2.3$ \\
\hline w;PDP1 $1 \varepsilon A /+; w ; P D P 1 \varepsilon B /+$ & $73.9(29)$ & $24.5 \pm 0.16$ & $78.1 \pm 11.7$ & $27.1 \pm 3.9$ \\
\hline 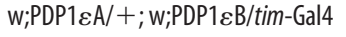 & $9.7(31)$ & $25.2 \pm 0.12$ & $24.5 \pm 5.9$ & $15.4 \pm 1.8$ \\
\hline 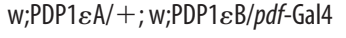 & $10.3(29)$ & $24.1 \pm 0.02$ & $27.2 \pm 2.3$ & $19.1 \pm 1.5$ \\
\hline
\end{tabular}

Period and power were calculated for all rhythmic animals as described in Materials and Methods. Activity was calculated for all flies that survived to the end of the experiment, as described in Materials and Methods. n.a., Not applicable.

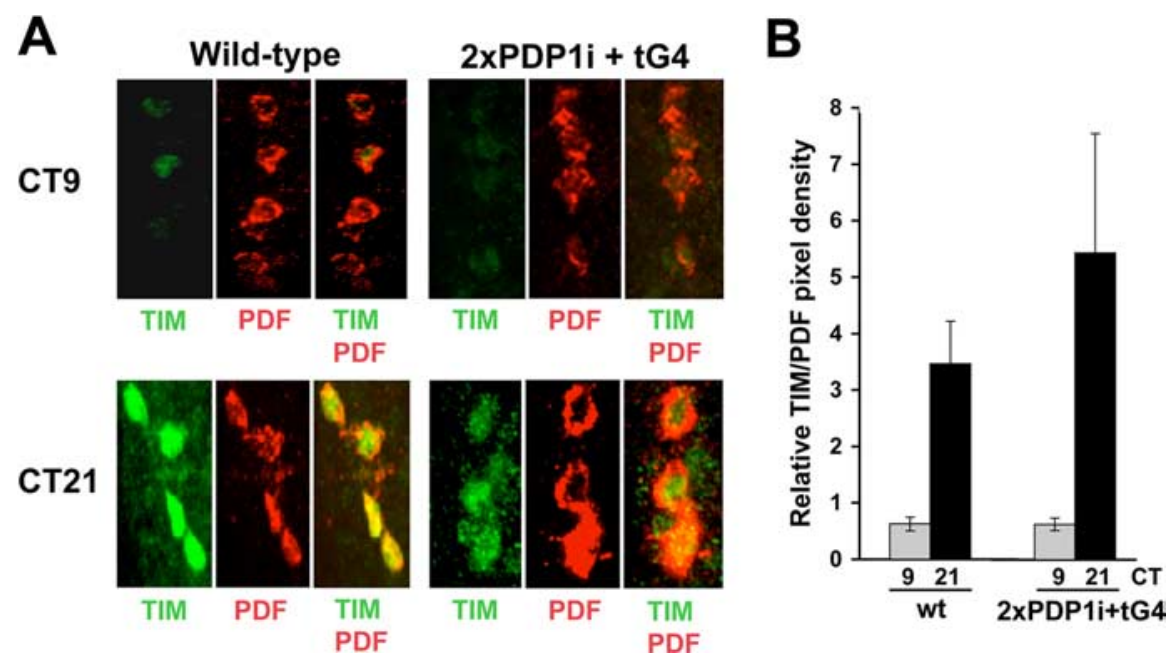

Figure 5. PDP1 RNAi does not disrupt TIM cycling in $s L N_{v} s$ during DD. $\boldsymbol{A}$, Brains dissected from wild-type and $w$;UAS-PDP1iA/ UAS-PDP1iB;timGal4/+ (2xPDP1i+tG4) flies collected during the second day of DD at CT9 and CT21 were probed with TIM and PDF antibodies. A representative $1 \mu \mathrm{m}$ optical section of $s L_{v} s$ is visualized for TIM (left panels), PDF (middle panels), or TIM plus PDF (right panels). TIM (green) and PDF (red) coimmunostaining is visualized as yellow. $\boldsymbol{B}$, TIM levels in control 2xPDP1i and $2 x P D P 1 i+t G 4$ flies was quantified as the ratio of TIM/PDF immunostaining. Staining in $S L N_{v} s$ was quantified from at least 12 brains (i.e., at least 4 brains from 3 independent experiments). wt, Wild type.
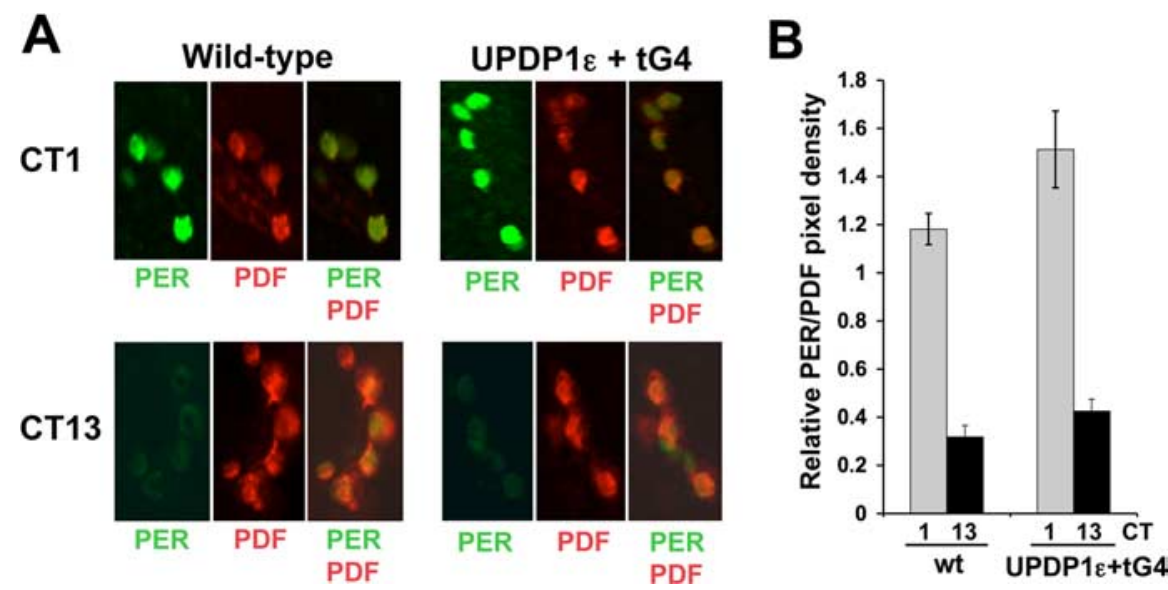

Figure 6. $\mathrm{PDP1} 1 \varepsilon$ overexpression does not disrupt PER cycling in $\mathrm{SLN}_{\mathrm{v}} \mathrm{s}$ during $\mathrm{DD}$. $\boldsymbol{A}$, Brains dissected from wild-type and w;UAS-PDP1 $1 \varepsilon /+$;UAS-PDP1 $1 /$ timGal4 (UPDP1 $\varepsilon+\mathrm{tG} 4$ ) flies collected during the second day of DD at CT1 and CT13 were probed with PER and PDF antibodies. A representative $1 \mu \mathrm{m}$ optical section of $s L_{v} s$ is visualized for PER (left panels), PDF (middle panels), or PER plus PDF (right panels). PER (green) and PDF (red) coimmunostaining is visualized as yellow. $\boldsymbol{B}$, PER immunostaining in wild-type (wt) and UPDP1 $\varepsilon+\mathrm{tG} 4$ flies was quantified as the ratio of PER/PDF staining. Staining in $s L N_{v} s$ was quantified from at least 12 brains (i.e., at least 4 brains from 3 independent experiments). 
in the $\mathrm{LN}_{\mathrm{v}} \mathrm{s}$ of flies expressing constant low levels of PDP1e (2xUAS-PDP1i+timGal4) is similar to that seen in $2 x U A S-$ PDP1i controls on day 5 of DD (supplemental Fig. $1 A, B$, available at www.jneurosci.org as supplemental material). Likewise, rhythmic PER immunofluorescence in the $\mathrm{LN}_{\mathrm{v}} \mathrm{s}$ of flies expressing constant high levels of PDP1 $\varepsilon$ (UPDP1 $1 \varepsilon+$ timGal4) is similar to that seen in UPDP1 $\varepsilon$ controls on day 5 of DD (supplemental Fig. $1 C, D$, available at www.jneurosci.org as supplemental material). These results demonstrate that the circadian oscillator continues to function at a time when behavioral rhythmicity is lost.

The loss of rhythmic behavior in 2xUAS-PDP1i+tim Gal4 and UPDP $1 \varepsilon+$ timGal4 flies suggests that PDP1 1 either controls rhythmic transcription of clock output genes or is necessary for the development of $s \mathrm{LN}_{\mathrm{v}} \mathrm{s}$. PDP1 $1 \varepsilon$, like other PDP1 isoforms, is expressed in embryos and larvae (Reddy et al., 2000). A Pdp1 null mutant, $P d p 1^{\mathrm{P} 205}$, causes developmental arrest during the larval stage, indicating that $P d p 1$ plays an essential role during larval development (Cyran et al., 2003; Reddy et al., 2006). We have shown that $\mathrm{sLN}_{\mathrm{v}} \mathrm{s}$ are present in flies expressing constant low or high levels of PDP1ع (Figs. 1, 3). However, it is possible that behavioral arrhythmicity results from missing or defective $\mathrm{sLN}_{\mathrm{v}}$ projections in 2xUAS-PDP1i+tim Gal4 and UPDP1 $\varepsilon+$ tim Gal4 flies. To investigate this possibility, projections from the $\mathrm{sLN}_{\mathrm{v}} \mathrm{s}$ were visualized using antisera against pigment dispersing factor (PDF), a neuropeptide that is necessary for behavioral rhythmicity (Renn et al., 1999).

The $\mathrm{sLN}_{\mathrm{v}} \mathrm{s}$ from all 2xUAS-PDP1i+timGal4 $(n=22$ brain hemispheres) and control 2xUAS-PDP1i $(n=8$ brain hemispheres) flies extend projections into the dorsal protocerebrum (supplemental Fig. $2 A, B$, available at www.jneurosci.org as supplemental material). Projections in 2xUAS-PDP1i+timGal4 flies were similar in morphology to those of $2 \mathrm{xUAS}-\mathrm{PDP} 1 \mathrm{i}$ controls in all but two cases: one projection split and then reunited, and one projection was overextended (supplemental Fig. 2C, available at www.jneurosci.org as supplemental material; and data not shown). Likewise, all projections from the $s \mathrm{LN}_{\mathrm{v}} \mathrm{s}$ of UPDP1 $\varepsilon+$ timGal4 ( $n=12$ brain hemispheres) and control UPDP1 $\varepsilon$ ( $n=8$ brain hemispheres) flies extend into the dorsal protocerebrum (supplemental Fig. 2D,E, available at www. jneurosci.org as supplemental material). UPDP1 $\varepsilon+$ tim Gal4 and control UPDP1 $\varepsilon \mathrm{sLN}_{\mathrm{v}}$ projections were similar in morphology with two exceptions: one UPDP1 $1 \varepsilon$ projection split and then reunited, and one UPDP $1 \varepsilon+$ timGal4 projection was overextended (supplemental Fig. $2 \mathrm{~F}$, available at www.jneurosci.org as supplemental material; and data not shown). These results demonstrate that constant low or high PDP1 $1 \varepsilon$ levels have little affect on the morphology of $\mathrm{sLN}_{\mathrm{v}}$ projections. Given that a single dorsally projecting $s \mathrm{sN}_{\mathrm{v}}$ is sufficient to mediate behavioral rhythms (Helfrich-Forster, 1998), these data argue that behavioral arrhythmicity in 2xUAS-PDP $1 \mathrm{i}+$ tim Gal4 and UPDP $1 \varepsilon+$ tim Gal 4 flies is attributable to the disruption of rhythmic output gene transcription.

\section{Discussion}

\section{PDP1 1 is not necessary for circadian oscillator function}

Transcriptional activation by PDP $1 \varepsilon$ and repression by VRI are thought to mediate Clk mRNA cycling (Cyran et al., 2003). Both VRI and PDP1e bind to V/P-boxes, and the ratio of VRI to PDP1 $\varepsilon$ binding is thought to control Clk mRNA cycling such that a high VRI/PDP1e ratio during the late day and early evening represses $C l k$ transcription and a low VRI/PDP1 $\varepsilon$ ratio from mid evening to early morning activates Clk transcription (Cyran et al., 2003). Because VRI levels peak during the early evening and
PDP1 $\varepsilon$ levels peak late in the evening (Cyran et al., 2003; Glossop et al., 2003), V/P-box binding and Clk transcription are directly related to the levels of VRI and PDP1 $\varepsilon$.

Because Pdp1 1 and vri are activated by CLK-CYC (Blau and Young, 1999; Cyran et al., 2003), it follows that Clk mRNA should be constantly low in a Clk or $c y c$ mutant because of low PDP1 $\varepsilon$ levels, yet constant high levels of Clk mRNA are seen (Glossop et al., 1999). This result might suggest that a low level of PDP1 $\varepsilon$ can fully activate $C l k$ when VRI levels are low (Cyran et al., 2003). The complete loss of all PDP1 isoforms in homozygous $P d p 1^{\mathrm{P} 205}$ larvae eliminates $C l k$ expression, indicating that PDP $1 \varepsilon$ activates Clk transcription (Cyran et al., 2003). However, the lack of Clk expression in $P d p 1^{\mathrm{P} 205}$ mutant larvae may also be attributable to developmental defects that damage or eliminate clock brain neurons, because $P d p 11^{\mathrm{P} 205}$ mutants are developmentally delayed and rarely survive beyond the third larval instar (Cyran et al., 2003). To avoid potential developmental problems arising from the loss of all PDP1 isoforms expressed in different tissues, we altered PDP1 $1 \varepsilon$ levels specifically in clock cells.

If rhythms in the ratio of VRI/PDP1 $\varepsilon$ are required for $C l k$ mRNA cycling, we reasoned that constant low or high levels of PDP $1 \varepsilon$ would chronically increase or decrease the VRI/PDP1 $1 \varepsilon$ ratio, respectively, and the resulting changes in Clk mRNA levels would disrupt Clk mRNA cycling. However, we found that neither constant low or constant high levels of PDP1 $\varepsilon$ disrupted $C l k$ mRNA cycling (Figs. 2A, 4A). When PDP1 1 levels in oscillator cells were constantly low, the amplitude of $C l k$ mRNA cycling was similar to that in timGal4 controls and wild-type flies (Fig. 2A) (Bae et al., 1998; Darlington et al., 1998). This conclusion is also supported by PDP1 $\varepsilon$ overexpression, in which $\sim 10$-fold higher levels of PDP1 $\varepsilon$ in oscillator cells alter neither the overall level of Clk mRNA nor the amplitude of Clk mRNA cycling (Fig. 4A). Comparable levels of Clk mRNA in flies expressing low or high levels of PDP1 1 in oscillator cells suggest that PDP1 $1 \varepsilon$ does not activate Clk transcription. However, we cannot discount some role for PDP $1 \varepsilon$ in activating Clk transcription because PDP $1 \varepsilon$ is not completely eliminated in 2xUAS-PDP $1 i+$ timGal4 flies, PDP1e may constantly be at saturating levels in 2xUASPDP $1 \varepsilon+$ timGal4 flies, and Clk mRNA is at lower levels in 2xUAS-PDP1i+timGal4 flies versus timGa14 controls just after dawn.

VRI is known to repress Clk transcription in vivo (Cyran et al., 2003; Glossop et al., 2003). Because Clk mRNA continues to cycle when PDP $1 \varepsilon$ levels are high or low, VRI levels would be predicted to cycle with peak levels coincident with the trough in Clk mRNA abundance. Indeed, VRI levels cycle robustly in both 2xUASPDP $1 \mathrm{i}+$ tim Gal4 and 2xUAS-PDP1 $\varepsilon+$ timGal4 flies (Figs. $2 B, C$, $4 B, C)$. This result not only reinforces the role of VRI as a $C l k$ repressor but provides additional evidence that the molecular feedback loops that underlie the circadian oscillator are operating normally.

\section{PDP1 $\varepsilon$ is required for behavioral rhythmicity}

The circadian oscillator is thought to control overt physiological, metabolic, and behavioral rhythms via rhythmically transcribed "output" genes. Microarray analyses of fly heads demonstrate that $>100$ transcripts are rhythmically expressed and that the vast majority of these transcripts represent clock output genes rather than oscillator components (Claridge-Chang et al., 2001; McDonald and Rosbash, 2001; Ceriani et al., 2002; Lin et al., 2002; Ueda et al., 2002; Wijnen et al., 2006). Some of these output transcripts appear to be directly controlled by CLK-CYC (McDonald and Rosbash, 2001), but their roles in regulating overt 


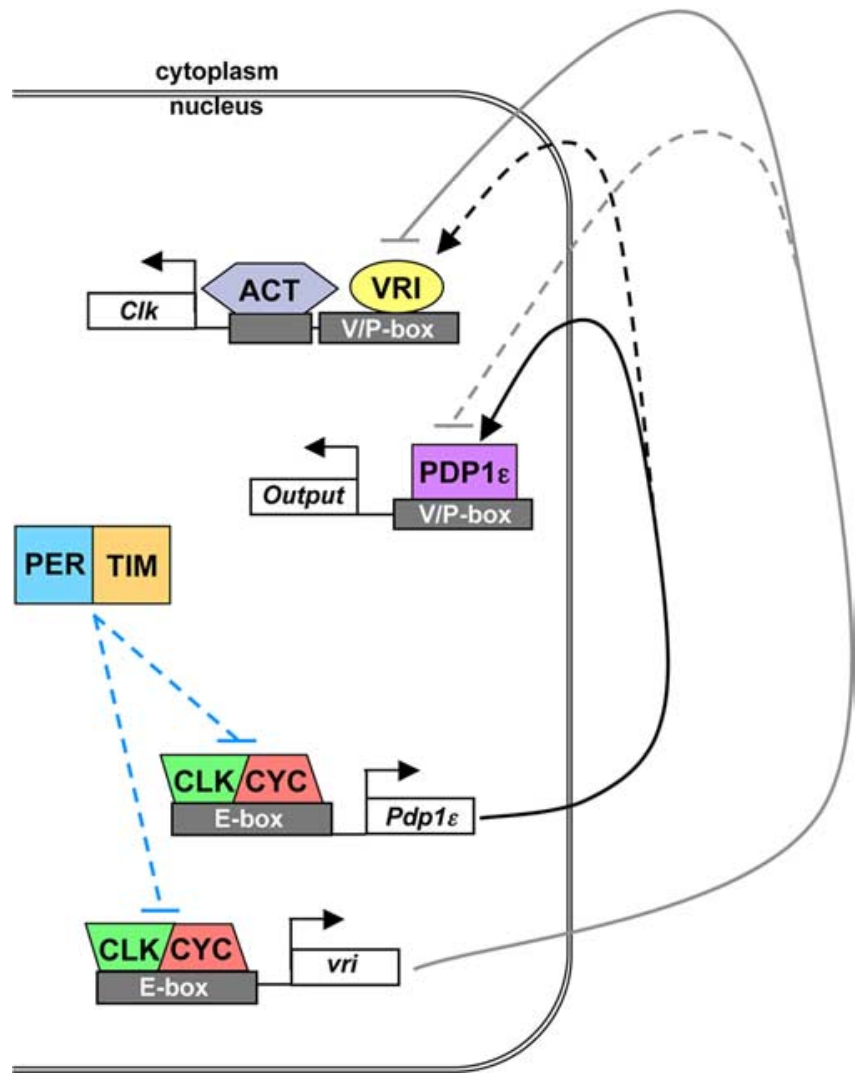

Figure 7. Model for the function of PDP1 $\varepsilon$. CLK-CYC heterodimers bind E-boxes to activate vri and $P d p 1 \varepsilon$ transcription. VRI accumulates in concert with its $m R N A$ and binds V/P-boxes to inhibit $C l k$ transcription (solid gray line). PDP1 $\varepsilon$ accumulates to peak levels several hours after VRI, activates output genes required for behavioral activity (solid black line), and possibly counteracts VRI inhibition (dashed black line). VRI may also contribute to rhythmic output gene transcription (dashed gray line). PER-TIM accumulates in parallel with PDP1 $\varepsilon$ and inhibits CLKCYC-dependent transcription of vri and Pdp1 $\varepsilon$ (dashed blue line), thereby removing VRI inhibition of $C l k$ transcription. An activator of $C l k$ transcription (ACT) is constantly present, thus accounting for high levels of $\mathrm{Clk} \mathrm{mRNA} \mathrm{in} C \mathrm{k}^{\mathrm{Jrk}}$ and $c y \mathrm{c}^{01}$ mutants and in wild-type flies when VRl is absent.

rhythms have not been characterized. A key property of clock output genes is that circadian oscillator function persists in their absence. Perhaps the best example of a clock output gene is the neuropeptide PDF which, when eliminated, has no effect on circadian oscillator function but abolishes locomotor activity rhythms (Renn et al., 1999). However, $p d f$ is not rhythmically transcribed, although its expression in $\mathrm{sLN}_{\mathrm{v}} \mathrm{s}$ is dependent on $\mathrm{Clk}$ and $c y c$ (Park et al., 2000). In fact, we do not know of any rhythmically expressed output genes that control specific physiological, metabolic, or behavioral rhythms.

Our analysis indicates that $P d p 1 \varepsilon$ is the first rhythmically expressed output gene that is required to mediate an overt circadian rhythm. Rhythmic transcription of $P d p 1 \varepsilon$ is directly controlled by CLK-CYC (Cyran et al., 2003), and constant low or high levels of PDP1 $\varepsilon$ have little effect on Clk mRNA cycling or circadian oscillator function in $\mathrm{sLN}_{\mathrm{v}} \mathrm{s}$ (Figs. 1-4) but severely impair behavioral rhythmicity (Table 1). The vast majority of flies expressing constant low or high levels of PDP1 1 in oscillator cells extend morphologically normal $\mathrm{sLN}_{\mathrm{v}}$ projections in at least one (and typically both) brain hemisphere, which indicates that behavioral arrhythmicity is not attributable to defects in $\mathrm{sLN}_{\mathrm{v}}$ development (supplemental Fig. 2, available at www.jneurosci.org as supplemental material). Because PDP $1 \varepsilon$ is a rhythmically expressed
bZIP transcriptional factor, we favor the possibility that PDP1 $\varepsilon$ mediates behavioral rhythms by rhythmically activating effector genes (Fig. 7). These second-order rhythmic transcripts may represent some of those identified in previous microarray studies, the abundance of which peaks near dawn (Claridge-Chang et al., 2001; McDonald and Rosbash, 2001; Ceriani et al., 2002; Lin et al., 2002; Ueda et al., 2002; Wijnen et al., 2006). In fact, it is possible that PDP1 $\varepsilon$ controls many second-order rhythmic transcripts and, consequently, other overt rhythms. Likewise, VRI may also contribute to overt rhythmicity by regulating clock output genes (Fig. 7). The extent to which PDP1 $\varepsilon$ and/or VRI control rhythmic transcripts that mediate physiological, metabolic, and behavioral rhythms awaits future study.

$\mathrm{PDP} 1 \varepsilon$ is related to three rhythmically expressed PAR domain-containing bZIP transcriptional activators in mammals: albumin gene site D-binding protein (DBP), thyroid embryonic factor, and hepatocyte leukemia factor (Wuarin and Schibler, 1990; Falvey et al., 1995; Fonjallaz et al., 1996). DBP knock-out mice show lower levels of locomotor activity and a shorter circadian period than wild-type animals, yet robust circadian oscillator function persists (Lopez-Molina et al., 1997). These results suggest that DBP functions to control clock output, consistent with the function of PDP $1 \varepsilon$ in flies. DBP binds to the same regulatory element as adenovirus E4 promoter ATF site-binding protein (E4BP4), a rhythmically expressed bZIP repressor that is related to VRI (Mitsui et al., 2001). Because E4BP4 is expressed in the opposite phase as DBP (Mitsui et al., 2001), sequential binding of E4BP4 and DBP could control rhythmic target gene transcription. Given that VRI and PDP1 $\varepsilon$ bind the same target sequence in flies, antagonistic binding of PAR domain transcription factors and their repressors could be a conserved mechanism for controlling rhythmic outputs.

\section{References}

Bae K, Lee C, Sidote D, Chuang KY, Edery I (1998) Circadian regulation of a Drosophila homolog of the mammalian Clock gene: PER and TIM function as positive regulators. Mol Cell Biol 18:6142-6151.

Blau J, Young MW (1999) Cycling vrille expression is required for a functional Drosophila clock. Cell 99:661-671.

Brand AH, Perrimon N (1993) Targeted gene expression as a means of altering cell fates and generating dominant phenotypes. Development 118:401-415.

Ceriani MF, Hogenesch JB, Yanovsky M, Panda S, Straume M, Kay SA (2002) Genome-wide expression analysis in Drosophila reveals genes controlling circadian behavior. J Neurosci 22:9305-9319.

Claridge-Chang A, Wijnen H, Naef F, Boothroyd C, Rajewsky N, Young MW (2001) Circadian regulation of gene expression systems in the Drosophila head. Neuron 32:657-671.

Cyran SA, Buchsbaum AM, Reddy KL, Lin MC, Glossop NR, Hardin PE, Young MW, Storti RV, Blau J (2003) vrille, Pdp1, and dClock form a second feedback loop in the Drosophila circadian clock. Cell 112:329-341.

Darlington TK, Wager-Smith K, Ceriani MF, Staknis D, Gekakis N, Steeves TD, Weitz CJ, Takahashi JS, Kay SA (1998) Closing the circadian loop: CLOCK-induced transcription of its own inhibitors per and tim. Science 280:1599-1603.

Emery P, So WV, Kaneko M, Hall JC, Rosbash M (1998) CRY, a Drosophila clock and light-regulated cryptochrome, is a major contributor to circadian rhythm resetting and photosensitivity. Cell 95:669-679.

Falvey E, Fleury-Olela F, Schibler U (1995) The rat hepatic leukemia factor (HLF) gene encodes two transcriptional activators with distinct circadian rhythms, tissue distributions and target preferences. EMBO J 14:4307-4317.

Fonjallaz P, Ossipow V, Wanner G, Schibler U (1996) The two PAR leucine zipper proteins, TEF and DBP, display similar circadian and tissuespecific expression, but have different target promoter preferences. EMBO J 15:351-362.

Glossop NR, Hardin PE (2002) Central and peripheral circadian oscillator mechanisms in flies and mammals. J Cell Sci 115:3369-3377. 
Glossop NR, Lyons LC, Hardin PE (1999) Interlocked feedback loops within the Drosophila circadian oscillator. Science 286:766-768.

Glossop NR, Houl JH, Zheng H, Ng FS, Dudek SM, Hardin PE (2003) VRILLE feeds back to control circadian transcription of Clock in the Drosophila circadian oscillator. Neuron 37:249-261.

Grumbling G, Strelets V (2006) FlyBase: anatomical data, images and queries. Nucleic Acids Res 34:D484-D488.

Hardin PE (2004) Transcription regulation within the circadian clock: the E-box and beyond. J Biol Rhythms 19:348-360.

Hardin PE (2005) The circadian timekeeping system of Drosophila. Curr Biol 15:R714-R722.

Hardin PE, Hall JC, Rosbash M (1990) Feedback of the Drosophila period gene product on circadian cycling of its messenger RNA levels. Nature 343:536-540.

Hazelrigg T, Levis R, Rubin GM (1984) Transformation of white locus DNA in Drosophila: dosage compensation, zeste interaction, and position effects. Cell 36:469-481.

Helfrich-Forster C (1998) Robust circadian rhythmicity of Drosophila melanogaster requires the presence of lateral neurons: a brain-behavioral study of disconnected mutants. J Comp Physiol A Neuroethol Sens Neural Behav Physiol 182:435-453.

Houl JH, Yu W, Dudek SM, Hardin PE (2006) Drosophila CLOCK is constitutively expressed in circadian oscillator and non-oscillator Cells. J Biol Rhythms 21:93-103.

Hunter-Ensor M, Ousley A, Sehgal A (1996) Regulation of the Drosophila protein timeless suggests a mechanism for resetting the circadian clock by light. Cell 84:677-685.

Konopka RJ, Benzer S (1971) Clock mutants of Drosophila melanogaster. Proc Natl Acad Sci USA 68:2112-2116.

Lee C, Bae K, Edery I (1999) PER and TIM inhibit the DNA binding activity of a Drosophila CLOCK-CYC/dBMAL1 heterodimer without disrupting formation of the heterodimer: a basis for circadian transcription. Mol Cell Biol 19:5316-5325.

Lin Y, Han M, Shimada B, Wang L, Gibler TM, Amarakone A, Awad TA, Stormo GD, Van Gelder RN, Taghert PH (2002) Influence of the perioddependent circadian clock on diurnal, circadian, and aperiodic gene expression in Drosophila melanogaster. Proc Natl Acad Sci USA 99:9562-9567.

Lopez-Molina L, Conquet F, Dubois-Dauphin M, Schibler U (1997) The DBP gene is expressed according to a circadian rhythm in the suprachiasmatic nucleus and influences circadian behavior. EMBO J 16:6762-6771.
McDonald MJ, Rosbash M (2001) Microarray analysis and organization of circadian gene expression in Drosophila. Cell 107:567-578.

Mitsui S, Yamaguchi S, Matsuo T, Ishida Y, Okamura H (2001) Antagonistic role of E4BP4 and PAR proteins in the circadian oscillatory mechanism. Genes Dev 15:995-1006.

Myers MP, Wager-Smith K, Rothenfluh-Hilfiker A, Young MW (1996) Light-induced degradation of TIMELESS and entrainment of the Drosophila circadian clock. Science 271:1736-1740.

Park JH, Helfrich-Forster C, Lee G, Liu L, Rosbash M, Hall JC (2000) Differential regulation of circadian pacemaker output by separate clock genes in Drosophila. Proc Natl Acad Sci USA 97:3608-3613.

Reddy KL, Wohlwill A, Dzitoeva S, Lin MH, Holbrook S, Storti RV (2000) The Drosophila PAR domain protein $1(P d p 1)$ gene encodes multiple differentially expressed mRNAs and proteins through the use of multiple enhancers and promoters. Dev Biol 224:401-414.

Reddy KL, Rovani MK, Wohlwill A, Katzen A, Storti RV (2006) The Drosophila Par domain protein I gene, Pdp1, is a regulator of larval growth, mitosis and endoreplication. Dev Biol 289:100-114.

Renn SC, Park JH, Rosbash M, Hall JC, Taghert PH (1999) A pdfneuropeptide gene mutation and ablation of PDF neurons each cause severe abnormalities of behavioral circadian rhythms in Drosophila. Cell 99:791-802.

Rutila JE, Suri V, Le M, So WV, Rosbash M, Hall JC (1998) CYCLE is a second bHLH-PAS clock protein essential for circadian rhythmicity and transcription of Drosophila period and timeless. Cell 93:805-814.

Sehgal A, Rothenfluh-Hilfiker A, Hunter-Ensor M, Chen Y, Myers MP, Young MW (1995) Rhythmic expression of timeless: a basis for promoting circadian cycles in period gene autoregulation. Science 270:808-810.

Tanoue S, Krishnan P, Krishnan B, Dryer SE, Hardin PE (2004) Circadian clocks in antennal neurons are necessary and sufficient for olfaction rhythms in Drosophila. Curr Biol 14:638-649.

Ueda HR, Matsumoto A, Kawamura M, Iino M, Tanimura T, Hashimoto S (2002) Genome-wide transcriptional orchestration of circadian rhythms in Drosophila. J Biol Chem 277:14048-14052.

Wijnen H, Naef F, Boothroyd C, Claridge-Chang A, Young MW (2006) Control of daily transcript oscillations in Drosophila by light and the circadian clock. PLoS Genet 2:e39.

Wuarin J, Schibler U (1990) Expression of the liver-enriched transcriptional activator protein DBP follows a stringent circadian rhythm. Cell 63:1257-1266.

Yu W, Zheng H, Houl JH, Dauwalder B, Hardin PE (2006) PER-dependent rhythms in CLK phosphorylation and E-box binding regulate circadian transcription. Genes Dev 20:723-733. 